\title{
VARIAÇÕES SAZONAIS NA SOBREVIVÊNCIA E PRODUÇÃo DE BIOMASSA DE Caesalpinia pyramidalis Tul. APÓS CORTE RASO E IMPLICAÇÕES PARA O MANEJO DA ESPÉCIE ${ }^{1}$
}

\author{
Joselma Maria de Figueirôa², Elcida de Lima Araújo ${ }^{3}$, Frans Germain Corneel Pareyn ${ }^{4}$, David F. Cutler ${ }^{4}$, \\ Peter Gasson ${ }^{4}$, Kleber Costa de Lima ${ }^{5}$ e Venézio Felipe dos Santos ${ }^{5}$
}

\begin{abstract}
RESUMO - A demanda de uso energético da vegetação da Caatinga tem gerado modificações nas paisagens e perda de diversidade biológica por insuficiência de informações sobre o manejo das espécies. Considerando o fato da estacionalidade climática ser um fator de influência na sobrevivência, ritmo biológico, rebrota e produtividade das plantas, neste estudo objetivou-se avaliar a influência da sazonalidade climática sobre a sobrevivência e a produção de biomassa de Caesalpinia pyramidalis Tul. (Caesalpiniaceae). Para tal, foram selecionados aleatoriamente 180 indivíduos de C. pyramidalis, sendo estes distribuídos em três blocos de 1 ha de caatinga (60 indivíduos por bloco). O delineamento experimental foi de blocos ao acaso com 6 tratamentos (sendo duas estações climáticas e três anos consecutivos de avaliação) para a avaliação da sobrevivência e com 4 tratamentos (duas estações climáticas e dois anos de medição) para a avaliação da biomassa aérea. As avaliações foram realizadas em duas estações: seca e chuvosa, sendo metade dos indivíduos de cada bloco $(n=30)$ submetidos à corte raso em cada uma das estações. Após o corte, a quantificação do peso fresco foi determinada em três componentes previamente definidos como lenha, estaca e graveto. As plantas foram monitoradas por 3 anos, sendo a sobrevivência anual registrada e a produção de biomassa da rebrota medida no último ano. A sobrevivência das plantas foi similar e elevada nos três anos, independente da estação climática. As plantas recém cortadas apresentaram elevado percentual de lenha e as rebrotas apresentaram elevado percentual de graveto. O tamanho inicial das plantas não esta relacionado à variação do peso fresco da rebrota. O estudo mostra que, apesar de ocorrer regeneração das plantas após corte raso, o tempo de três anos não é suficiente para recuperação da produção dos produtos madeireiros em C. pyramidalis, sendo necessário um tempo de repouso maior para um novo ciclo de corte, visando manter a produção da espécie para atender a demanda energética da população rural.
\end{abstract}

Palavras chave: Biomassa, lenha, estaca, graveto, caatinga.

\section{SEASONAL VARIATIONS IN THE SURVIVAL AND BIOMASS PRODUCTION OF Caesalpinia pyramidalis Tul. AFTER COPPICING AND IMPLICATIONS FOR MANAGEMENT OF THE SPECIES}

\begin{abstract}
The demand for wood fuel from caatinga vegetation has caused landscape modifications and loss of biodiversity due to insufficient information on species management. As seasonal climate influences plant survival, biological rhythm, regrowth and productivity, this study aimed to evaluate the influence of
\end{abstract}

\footnotetext{
${ }^{1}$ Recebido em 30.05.2007 e aceito para publicação em 22.08.2008.

${ }^{2}$ Faculdade de Ciencias Aplicadas José Lacerda Filho (FAJOLCA). Recife-PE. E-mail: <joselmafigueiroa@ fajolca.edu.br>.

${ }^{3}$ Departamento de Biologia da Universidade Federal Rural de Pernambuco (UFPE). E-mail: <elcida@ db.ufrpe.br>.

${ }^{4}$ Royal Botanic Gardens Kew.

${ }^{5}$ Empresa Pernambucana de Pesquisa Agropecuária (IPA/PE). E-mail:<venezio@ipa.br>.
} 


\begin{abstract}
seasonality on the biological rhythm and biomass production of Caesalpinia pyramidalis Tul. (Caesalpiniaceae).A total of 180 individuals of $\boldsymbol{C}$. pyramidalis were randomly selected, distributed in three blocks of 1 ha of caatinga (60 trees each). The experiment was arranged in a randomized block design with six treatments (two seasons and three consecutive evaluation years) for survival and four treatments (two seasons and two measurement years) for aerial biomass production. Measurements were carried out in two seasons: dry and wet, with half of the number of individuals in each block $(n=30)$ being coppiced in each season. After cutting, fresh weight was determined for three components: fuel wood, posts and "twigs and leaves". The trees were monitored for three years, their survival monitored annually and regrowth biomass production measured in the last year. The survival of the trees was high and similar during the three years, independent of the season in which they were coppiced. When originally cut, the trees provided a high percentage of fuel wood and the regrowth provided a high percentage of "twigs and leaves". The original size of the trees does not explain the variation in regrowth fresh weight. The study shows that, despite sprouting after coppicing, three years are not sufficient to provide adequate wood production in $\boldsymbol{C}$. pyramidalis. A longer fallow period is necessary to guarantee sufficient growth to meet wood fuel demand of the rural population.
\end{abstract}

Key words: Biomass, fuel wood, post, "twigs and leaves", caatinga

\section{INTRODUÇÃO}

A sazonalidade climática é um fator abiótico de influência na sobrevivência, ritmo fenológico, produtividade e capacidade de rebrota das plantas de diferentes formações vegetacionais (MORELLATO et al., 2000; ANDREIS et al., 2003; BORCHERT et al., 2004; TEWARI et al., 2004; McCRAY et al., 2005; ARAÚJO et al., 2005; MANTOVANI et al., 2005), sobretudo aquelas consideradas secas e marcadas por forte estacionalidade (LINAREZ-PALOMINO e ALVAREZ, 2005; MONTEIRO et al., 2005; ARAÚJO et al., 2007). No Brasil, a Caatinga ocupa as paisagens da maior parte da Região Nordeste do país (ARAÚJO et al., 1995; ARAÚJO e TABARELLI, 2002; MONTEIRO et al., 2005; FIGUEIRÔA et al., 2006) e constitui um bom exemplo de vegetação com clima estacional, em que a sazonalidade climática é admitida como fator de estresse, funcionando como força seletiva modeladora da dinâmica e regeneração da vegetação (ARAÚJO e FERRAZ, 2003; ARAÚJO, 2005; ARAÚJO et al., 2007).

A maioria da população que vive nessa região apresenta baixa renda per capita e faz uso da vegetação como recurso para subsistência. Portanto, a relação homem-recurso também é influenciada pela característica sazonal do ambiente (TEWARI et al., 2004; McCRAY et al., 2005; ARAÚJO et al., 2007). No entanto, informações sobre o manejo adequado das diferentes espécies utilizadas geralmente não são conhecidas e nem acessíveis para a população local. Uma das principais formas de uso da vegetação da caatinga é o corte da

R. Árvore, Viçosa-MG, v.32, n.6, p.1041-1049, 2008 madeira para lenha e carvão (SAMPAIO et al., 1998; VÍRGINIO e PAREYN, 2002; FRANCELINO et al., 2003; ANDRADE et al., 2005; FIGUEIRÔA et al., 2005; FIGUEIRÔA et al., 2006; ARAÚJO et al., 2007; SANTOS et al., 2008), o que também ocorre em outras regiões secas do mundo (MIRSA et al., 1995; McLAREN e McDONALD, 2003; LUOGA et al., 2004; TEWARI et al., 2004; McCRAY et al., 2005; VALE e FELFILI, 2005). Contudo, faltam informações sobre o tratamento e época de corte que causem menos impacto sobre a capacidade de regeneração e recuperação da produtividade das diferentes espécies exploradas.

Caesalpinia pyramidalis Tul. é uma das espécies da Caatinga explorada pela população local como fonte de energia doméstica (lenha e carvão vegetal), fins medicinais e forrageiro (FRANCELINO et al., 2003; FIGUEIRÔA et al., 2005, 2006; SANTOS et al., 2008), sendo de ampla distribuição no Nordeste, mas endêmica da vegetação da Caatinga (MONTEIRO et al., 2005; FIGUEIRÔA et al., 2005). Os estudos realizados nessa formação vegetacional têm apontado que $C$. pyramidalis apresenta valores elevados de frequiência e de densidade nas unidades utilizadas para amostragem da vegetação (ARAÚJO et al., 1995; ALCOFORADO-FILHO et al., 2003; AMORIM et al., 2005; SANTANAe SOUTO, 2006), indicando que a população tende a apresentar padrão de distribuição espacial regular.

Os porcentuais de sobrevivência e de rebrota das espécies da Caatinga, quando submetidas a diferentes tipos de corte, foram estudados em poucas áreas do Nordeste brasileiro e mostraram que muitas espécies 
desse ambiente seco, como C. pyramidalis, Croton sonderianus, Mimosa tenuiflora etc., em geral apresentam capacidade de rebrota após o corte (SAMPAIO et al., 1998; FIGUEIRÔA et al., 2006), o que também ocorre em outras áreas de ambientes semi-áridos do mundo (MCLAREN e MCDONALD, 2003; LUOGA et al., 2004; TEWARI et al., 2004; McCRAY et al., 2005).

Na prática do corte raso de plantas da Caatinga, é usual ser mantida a base do caule da planta, sendo esperado que as reservas existentes nessa base sejam suficientes para favorecer a rebrota das plantas que sobreviverem. No entanto, não existem informações sobre a sobrevivência de plantas cortadas após a influência de estações chuvosas e secas consecutivas. Uma planta cortada pode produzir rebrota logo após o corte, mas esta pode morrer com a chegada da estação seca, e as reservas existentes na base do caule cortado podem não ser suficientes para favorecer a produção de novas rebrotas, sendo a morte da planta registrada mais tardiamente. Além disso, não existem informações sobre a influência do tamanho inicial do caule da planta sobre a produção de massa aérea após o tratamento de corte em plantas da Caatinga, visando estabelecer estratégias de conservação e manejo sustentável. Assim, este estudo objetivou avaliar se existe diferença na sobrevivência e produção de biomassa aérea de plantas de $C$. pyramidalis após corte raso nas estações chuvosa e seca, num intervalo de 3 anos, bem como identificar a existência de correlação entre tamanho inicial do caule e potencial de recuperação de massa aérea após o corte raso.

\section{MATERIAL E MÉTODOS}

O estudo foi desenvolvido em três áreas semiáridas do Estado de Pernambuco, sendo uma localizada na Estação Experimental da Empresa Pernambucana de Pesquisa Agropecuária - IPA, no Município de Serra Talhada, PE (0755'46.4' S 38 17'20.0”'W), com 80 ha; e as outras duas localizadas no Município de Sertânia, uma delas em terreno pertencente à mesma empresa mencionada acima (08 $04^{\circ}$ '02,7' $\mathrm{S} 37^{\circ} 12$ ' 33,1 ' 'W), com 40 ha, e a outra na comunidade de Caroalina ( $08^{\circ} 15^{\prime} 33,0^{\prime \prime} \mathrm{S}$ $\left.37^{\circ} 32^{\prime} 52,1^{\prime \prime} \mathrm{W}\right)$, com 20 ha.

As três áreas apresentavam solo de textura francoarenosa e clima estacional, com estação chuvosa indo de janeiro a maio. O clima da região é do tipo BShs', de acordo com a classificação de Köppen (ANDRADELIMA, 1981). A precipitação média anual foi de 626 mm em Serra Talhada, variando de 557 mm na estação chuvosa e 69 mm na estação seca e de 483 mm em Sertânia, variando de $428 \mathrm{~mm}$ na estação chuvosa e $55 \mathrm{~mm}$ na estação seca (FIGUEIRÔA et al., 2006). A fisionomia da vegetação das três áreas era do tipo Caatinga arbustiva arbórea e apresentava elevada riqueza de espécies pertencentes às famílias Leguminosae e Euphorbiaceae, como registrado para muitas áreas de Caatinga (ARAÚJO et al., 1995; FERRAZ et al., 2003; AMORIM et al., 2005; FERRAZ et al., 2006; ARAÚJO et al., 2007; SANTOS et al., 2008).

O delineamento experimental foi de blocos ao acaso com seis tratamentos (sendo duas estações climáticas e três anos consecutivos de avaliação) para a avaliação da sobrevivência e com quatro tratamentos (duas estações climáticas e dois anos de medição) para a avaliação da biomassa aérea. Em cada área, um bloco amostral de 1 ha foi selecionado para o estudo, sendo inicialmente realizada amostragem-piloto para determinar um número mínimo de plantas que fosse representativo para o estudo da população de $C$. pyramidalis. Na amostragem-piloto, foram selecionadas 100 indivíduos da população e mensurada a circunferência na base do caule. O número mínimo de plantas para representar a população de $C$. pyramidalis foi calculado pela fórmula para amostragem infinita (SNEDECOR e COCHRAN 1967), adotando-se um erro de $10 \%$ da média amostral e um nível de confiança de $95 \%$ de probabilidade, o qual resultou em um número mínimo de 20 plantas. Para aumentar a inclusão da variabilidade existente na população, o número mínimo de indivíduos foi acrescido em 50\%, resultando na seleção de 30 plantas por bloco amostral e por estação climática, totalizando 180 plantas.

Para avaliar a influência da sazonalidade climática na sobrevivência e na capacidade de recuperação da massa aérea após o corte, em cada bloco amostral, 30 plantas nas estações chuvosa (março/2002) e seca (setembro/ 2002) foram marcadas com plaquetas de alumínio presas com o auxílio de um arame; numeradas; mensuradas quanto à circunferência na base do caule $(\mathrm{cm})$; e submetidas a corte raso. A distância entre as plantas marcadas variou de 8 a 20 m, a fim de evitar que as copas se tocassem. Ramos férteis das plantas de cada área foram coletados para confirmação da identificação taxonômica da espécie. As exsicatas do material coletado encontram-se depositadas no Herbário Dárdano de Andrade Lima (IPA) (números IPA - 66039; IPA - 63361).

R. Árvore, Viçosa-MG, v.32, n.6, p.1041-1049, 2008 
A parte aérea cortada (massa verde) foi separada em três classes de produtos madeireiros (estaca, lenha e graveto) e devidamente pesada em balanças manuais, marca Caudura números 58117 e 3638, com capacidade máxima de $150 \mathrm{~kg} \mathrm{e} 25 \mathrm{~kg}$, respectivamente, e marca Tríplice escala JB, modelo 007 com capacidade máxima de 1,610 $\mathrm{kg}$. O produto-estaca correspondeu à madeira (fuste) com circunferência $\geq a 30 \mathrm{~cm}$; o produto lenha correspondeu à madeira com circunferência $\geq \mathrm{a} 6 \mathrm{~cm}$ e $<30 \mathrm{~cm}$, e o produto graveto correspondeu à madeira que apresentava circunferência menor que $6 \mathrm{~cm}$. O tamanho da circunferência para estaca, lenha e graveto foi adotado baseado no indicativo de uso do homem do campo para exploração de lenha e produção de carvão na região semi-árida (ZAKIA et al., 1990; FRANCELINO et al., 2003).

A sobrevivência dos indivíduos cortados em cada estação climática por bloco amostral foi avaliada pela ocorrência de rebrota na região do corte durante os três anos consecutivos à aplicação do tratamento (2003, 2004 e 2005). A sobrevivência de cada ano foi calculada com base no número inicial de indivíduos. Plantas que não apresentaram evidências de rebrota foram consideradas mortas.

Para a avaliação da recuperação da massa verde aérea, após três anos da aplicação do corte raso (estações chuvosa e seca de 2005), as rebrotas das plantas foram submetidas à nova intervenção de corte, sendo a massa aérea da rebrota separada quanto aos produtos estaca, lenha e graveto, os quais foram pesados em balanças manuais.

Os porcentuais médios de sobrevivência registrados durante os três anos de monitoramento foram comparados por análise de variância (ANOVA) (ZAR, 1996). A recuperação da massa aérea (estaca, lenha e graveto) foi avaliada por análise de co-variância (ANCOVA) (ZAR, 1996), sendo os dados previamente transformados na escala $\log _{10}(X+1)$ e as médias ajustadas pela covariável circunferência na base do caule. A significância das interações foi avaliada pelo teste $\mathrm{F}$ a $5 \%$ de probabilidade, e as diferenças entre as médias de sobrevivência e de produção de massa aérea foram comparadas pelo teste de Tukey a $5 \%$ de probabilidade. A relação entre o tamanho inicial da circunferência da planta e a produção de massa aérea em 2002 e 2005 foi avaliada pela análise de regressão linear (ZAR, 1996). Apenas as análises de regressão significativa foram apresentadas. As análises foram feitas através dos Programas SAEG (EUCLIDES, 1983) e Statistix 7.0 (ANALYTICAL SOFTWARE, 2000).

R. Árvore, Viçosa-MG, v.32, n.6, p.1041-1049, 2008

\section{RESULTADOS E DISCUSSÃO}

A sobrevivência dos indivíduos de Caesalpinia pyramidalis submetidos a corte raso na estação chuvosa variou de 98,9 a 96,0\%, enquanto na estação seca foi de $100 \%$ durante os três anos de monitoramento, não apresentando diferenças entre estações climáticas e entre anos (Figura 1).

A elevada sobrevivência de $C$. pyramidadalis nas estações chuvosa e seca após um ano de corte já havia sido registrada por Figueirôa et al. (2006), levando aos autores concluírem que o manejo da espécie por corte raso não era um fator de declínio populacional. Todavia, estudos sobre fenologia e dinâmica regenerativa de plantas da Caatinga têm evidenciado que essa espécie reflete a influência das variações sazonais em sua fenologia e dinâmica populacional (SAMPAIO et al., 1998; FIGUEIRÔA et al., 2005; MONTEIRO et al., 2005; ANDRADE et al., 2005; ARAÚJO et al., 2005). Baseado nessas evidências, esperava-se encontrar diferença significativa na sobrevivência de plantas cortadas de C. pyramidalis, após um período maior de monitoramento, diferentemente do registrado neste estudo. Logo, esses resultados confirmam que o manejo da espécie através do tratamento corte raso, nas diferentes estações climáticas, não é um fator que leve à morte da planta numa curta série temporal.

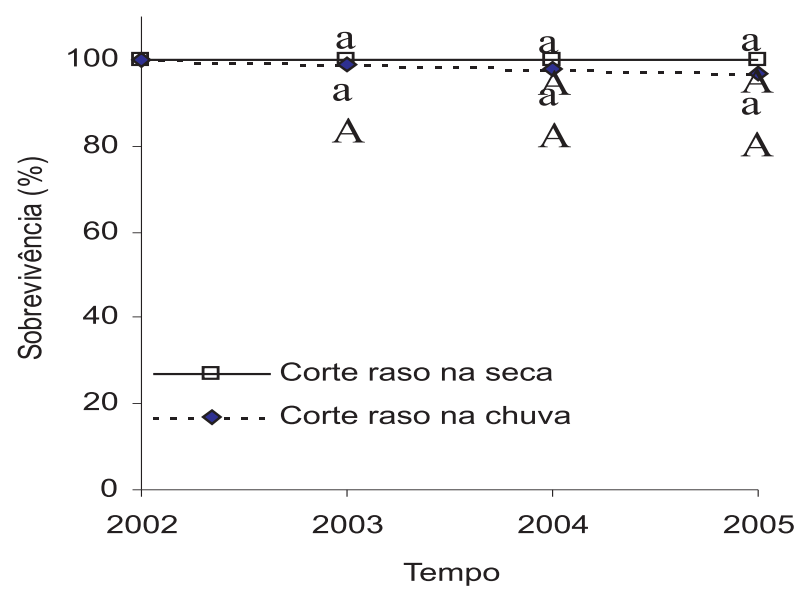

Figura 1 - Porcentual médio de sobrevivência de Caesalpinia pyramidalis após o corte raso durante três anos. Médias seguidas de mesmaletraminúsculaentreestações climáticas de cada ano e mesma letra maiúscula entre os anos não diferem pelo teste de Tukey $(\mathrm{P}=0,05)$.

Figure 1-Mean percentage of survival of Caesalpinia pyramidalis trees after coppicing during three years. Values followed by the same letter are not significantly different using the Tukey test $(P=0.05)$. 
Em 2002, o peso fresco total da parte aérea dos indivíduos de $C$. pyramidalis submetidas a corte raso foi de $3.240,5 \mathrm{~kg}$ na estação chuvosa e $3.572,1$ kg na estação seca. Na estação chuvosa, a média do peso das plantas em termos de aproveitamento para os produtos estaca, lenha e graveto, respectivamente, foi distribuída em 12,4; 61,0; e 26,6\%, enquanto na estação seca foi de 14,0;62,1; e 23,9\%, respectivamente. Após três anos do corte, as rebrotas das plantas apresentaram diferenças significativas na produção de estaca, lenha e graveto, em relação à biomassa original, independentemente da estação climática (Tabela 1). O peso de matéria fresca total da biomassa aérea das plantas cortadas em 2005 foi de $256,5 \mathrm{~kg}$ na estaca chuvosa e de $281,55 \mathrm{~kg}$ na estação seca. Em média, os indivíduos cortados na estação chuvosa de 2005 produziram apenas $4,3 \%$ de lenha e $95,7 \%$ de graveto, não havendo produção de estaca. Tendência semelhante foi registrada nas plantas cortadas na estação seca, que em média produziram
$5,6 \%$ de lenha, $94,4 \%$ de graveto e $0 \%$ de estaca.

A alteração registrada nas proporções dos produtos madeireiros das plantas cortadas mostra que $C$. pyramidalis apresenta leve tendência de produzir um pouco mais de lenha se as plantas forem cortadas na estação seca, bem como indicam que o tempo de três anos não é suficiente para que plantas dessa espécie apresentem recuperação quanto a produção de lenha e estaca.

Apesar de a sazonalidade climática ser um fator de influência na produtividade das plantas de diferentes áreas do mundo (MORELLATO et al., 2000; ANDREIS et al., 2003; ARAÚJO e FERRAZ, 2003; TEWARI et al., 2004; McCRAY et al., 2005; ARAÚJO 2005; MANTOVANI et al., 2005; ARAÚJO et al., 2007), no intervalo de tempo deste estudo não foram constatadas diferenças entre estações climáticas, quanto à recuperação da produção de biomassa aérea das plantas cortadas.

Tabela 1 - Média e respectivo erro-padrão dos pesos frescos ( $\mathrm{kg}$ ) dos produtos: estaca, lenha, graveto e peso total de Caesalpinia pyramidalis em 2002 (no ato do primeiro corte) e 2005 (corte da rebrota). Letras distintas seguidas na comparação de médias de um mesmo produto indicam diferença pelo teste de Tukey $(\mathrm{P}=0,05)$

Table 1 - Mean and respective standard error of fresh weight (kg) of posts, fuel wood and twigs and leaves and total weight of Caesalpinia pyramidalis in 2002 (first cut) and 2005 (regrowth cut). Distinct letters for the same products indicate difference using the Tukey test $(P=0.05)$

\begin{tabular}{|c|c|c|c|c|c|c|c|c|c|}
\hline \multirow[b]{2}{*}{ Tratamentos } & & \multicolumn{8}{|c|}{ Produtos Madeireiros } \\
\hline & & \multicolumn{2}{|c|}{ Estaca } & \multicolumn{2}{|c|}{ Lenha } & \multicolumn{2}{|c|}{ Graveto } & \multicolumn{2}{|c|}{ Peso Total } \\
\hline \multicolumn{10}{|c|}{ Anos/estações chuvosas } \\
\hline 2002 & & \multicolumn{2}{|c|}{$4,60 \pm 0,28$} & \multicolumn{2}{|c|}{$22,88 \pm 1,36$} & \multicolumn{2}{|c|}{$9,95 \pm 1,33$} & \multicolumn{2}{|c|}{$37,42 \pm 2,16$} \\
\hline 2005 & & \multicolumn{2}{|c|}{$0,00 \pm 0,00$} & \multicolumn{2}{|c|}{$0,13 \pm 0,05$} & \multicolumn{2}{|c|}{$2,91 \pm 0,26$} & \multicolumn{2}{|c|}{$3,06 \pm 0,29$} \\
\hline \multicolumn{10}{|l|}{ Anos/estações secas } \\
\hline 2002 & & \multicolumn{2}{|c|}{$5,41 \pm 0,47$} & \multicolumn{2}{|c|}{$23,83 \pm 0,15$} & \multicolumn{2}{|c|}{$9,13 \pm 1,27$} & \multicolumn{2}{|c|}{$38,37 \pm 1,72$} \\
\hline 2005 & & \multicolumn{2}{|c|}{$0,00 \pm 0,00$} & \multicolumn{2}{|c|}{$0,17 \pm 0,09$} & \multicolumn{2}{|c|}{$2,88 \pm 0,25$} & \multicolumn{2}{|c|}{$3,06 \pm 0,29$} \\
\hline \multicolumn{10}{|l|}{ Estações } \\
\hline Chuvosa & & \multicolumn{2}{|c|}{$2,30 \pm 0,14 \mathrm{a}$} & \multicolumn{2}{|c|}{$11,45 \pm 0,70 \mathrm{a}$} & \multicolumn{2}{|c|}{$5,01 \pm 0,80 \mathrm{a}$} & \multicolumn{2}{|c|}{$18,78 \pm 1,22 \mathrm{a}$} \\
\hline Seca & & \multirow{2}{*}{\multicolumn{2}{|c|}{$2,70 \pm 0,24 \mathrm{a}$}} & \multicolumn{2}{|c|}{$11,91 \pm 0,82 \mathrm{a}$} & \multicolumn{2}{|c|}{$4,57 \pm 0,75 \mathrm{a}$} & \multicolumn{2}{|c|}{$19,19 \pm 1,00 \mathrm{a}$} \\
\hline \multicolumn{9}{|l|}{ Anos } & \\
\hline 2002 & & 5,00 &, $38 \mathrm{a}$ & $23,35=$ & 40a & 9,5 & $30 \mathrm{a}$ & 37,8 & $1,94 \mathrm{a}$ \\
\hline 2005 & & 0,00 &, $00 \mathrm{~b}$ & $0,15+$ & & 2,9 & $26 \mathrm{~b}$ & 3,0 &, $23 b$ \\
\hline Fonte de variação & $\mathrm{gl}$ & $F$ & $P$ & $F$ & $P$ & $F$ & $P$ & $F$ & $P$ \\
\hline Blocos & 2 & 0,53 & 0,59 & 1,59 & 0,20 & 0,28 & 0,75 & 2,53 & 0,88 \\
\hline Anos & 1 & 2180,09 & 0,00 & 3198,49 & 0,00 & 4,00 & 0,05 & 1049,27 & 0,00 \\
\hline Estações & 1 & 6,35 & 0,01 & 0,29 & 0,59 & 0,36 & 0,55 & 3,55 & 0,06 \\
\hline Anos $x$ estações & 1 & 3,51 & 0,06 & 1,23 & 0,27 & 0,01 & 0,99 & 0,90 & 0,34 \\
\hline Resíduo & 353 & & & & & & & & \\
\hline
\end{tabular}


A retirada da vegetação para consumo de lenha e carvão e a capacidade de rebrota das plantas após o corte têm sido estudadas em diferentes florestas do mundo (MIRSA et al., 1995; SAMPAIO et al., 1998; NEGREROS-CASTILLO e HALL, 2000; McLAREN e McDONALD, 2003; FRANCELINO et al., 2003; LUOGA et al., 2004; McCRAY et al., 2005; VALE e FELFILI, 2005; FIGUEIRÔA et al., 2006), evidenciando o potencial de resiliência delas. Algumas espécies, como Mimosa tenuiflora e Mimosa ophthalmocentra, apresentaram melhor resposta de rebrota se cortadas em estação climática específica (estação seca) e outras não, como Caesalpinia pyramidalis e Croton sonderiaus

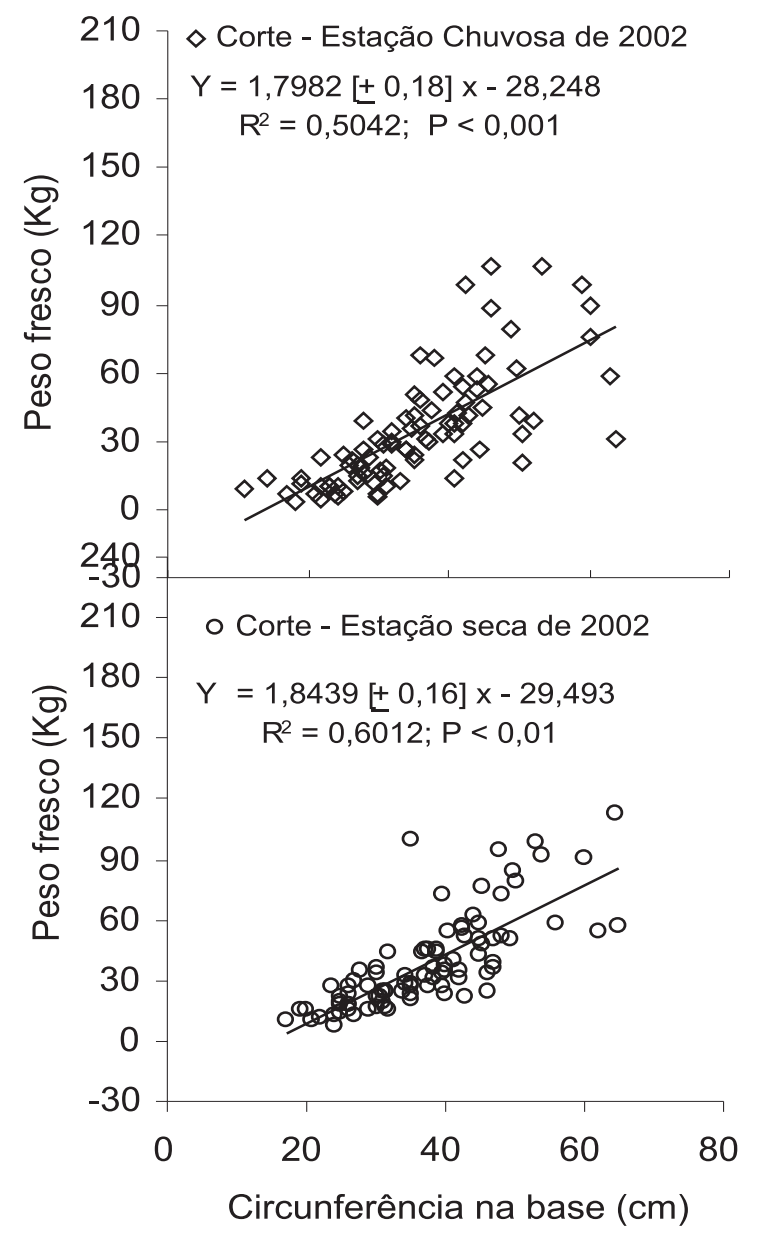

(FIGUEIRÔA et al., 2006). Mesmo assim, freqüentemente tem sido constatado que a exploração da vegetação para lenha e carvão, muitas vezes, leva ao declínio populacional da espécie explorada, podendo ocorrer perda da diversidade biológica. Porém, os resultados registrados neste trabalho indicaram que a perda de diversidade de espécies lenhosas pelo uso energético da vegetação nativa depende da espécie utilizada, pois o corte raso aplicado em $C$. pyramidalis, em qualquer estação climática, não reduziu sua capacidade de sobrevivência, o que já havia sido registrado para essa mesma espécie por Sampaio et al. (1998) em Serra Talhada e por Figueirôa et al. (2006) em Sertânia e em Serra Talhada.

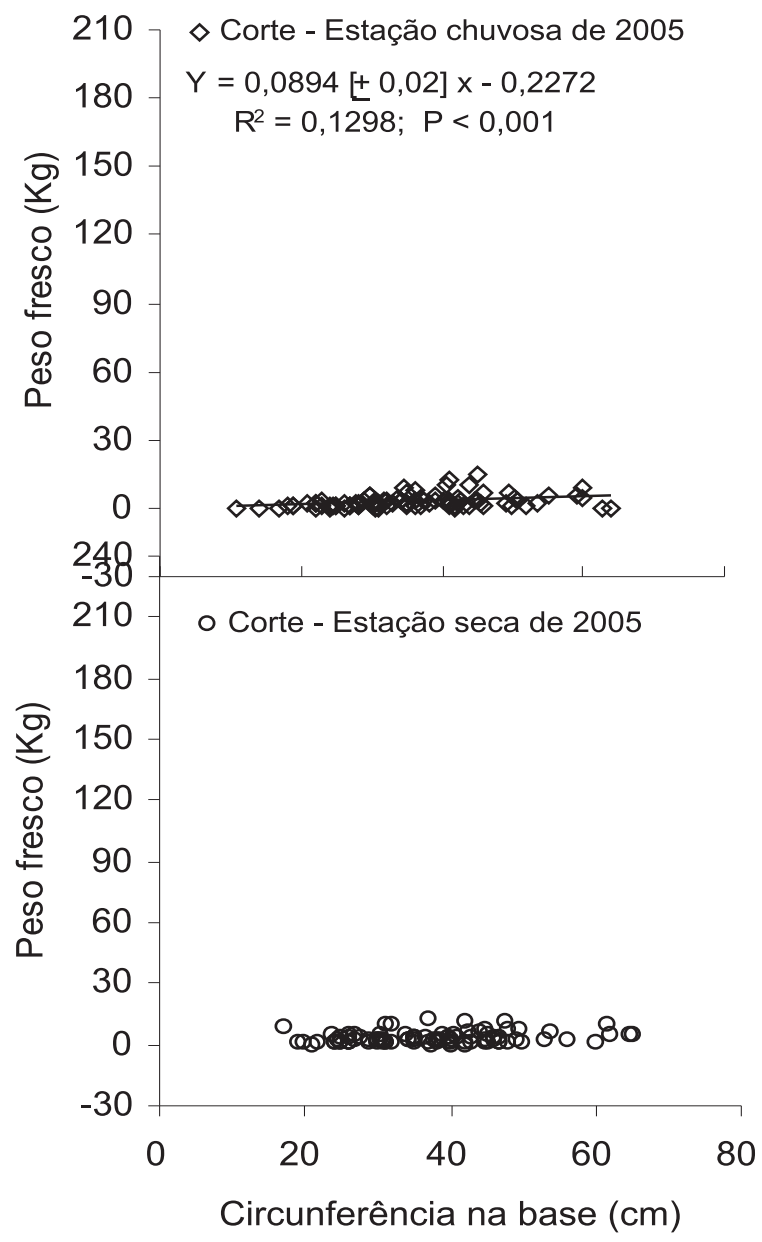

Figura 2 - Relação entre a circunferência da base da planta e o peso fresco total da parte aérea em Caesalpinia pyramidalis, no ato do corte raso nas estações chuvosa e seca do ano de 2002 e três anos após o primeiro corte. Somente foram apresentadas retas de regressão linear que foram significativas.

Figure 2 - Relationship between the tree basal circumference and the fresh weight of aerial biomass of Caesalpinia pyramidalis in wet and dry season in 2002 and after three years. Only linear regression lines that are significant are shown.

R. Árvore, Viçosa-MG, v.32, n.6, p.1041-1049, 2008 
O histórico do uso energético da vegetação da Caatinga indica que a prática da retirada da vegetação para lenha e carvão é uma realidade que deverá ser mantida nas próximas décadas pela população rural (VÍRGINIO et al., 2002; FRANCELINO et al., 2003), mas se a exploração for feita de forma adequada, isto é, utilizando-se apenas espécies que tenham capacidade de suportar o estresse da exploração e de tempo conhecido para recuperação das da biomassa explorada, a perda local de diversidade biológica das espécies utilizadas e os danos à regeneração natural da floresta poderão ser minimizados.

A circunferência basal das plantas nas estações chuvosa e seca, respectivamente, determinou 50 e $60 \%$ da variação do peso fresco da parte área no ato da aplicação do primeiro corte raso (2002). Todavia, após três anos da aplicação do tratamento a circunferência inicial da base das plantas passou a determinar apenas $12 \%$ da variação do peso da rebrota na estação chuvosa. $\mathrm{Na}$ estação seca, a relação peso de matéria fresca e tamanho inicial do caule não foi significativa (Figura 2). Isso mostra que a variação da massa aérea da planta, após tratamento de corte, independe de seu tamanho inicial de circunferência. Logo, no manejo da espécie a variável circunferência inicial do caule não permite projetar quantitativamente a produção dos produtos madeireiros (estaca, lenha e graveto) de plantas cortadas após três anos. Esse resultado difere do que vem sendo registrado para outras espécies de floresta seca do mundo, pois, em média, plantas com maior tamanho de caule têm possibilitado maior produção de rebrota de grandes tamanhos, em comparação com plantas de menores tamanhos de caule (McLAREN e McDONALD, 2003; LUOGA et al., 2004; NORDH e VERWIJST, 2004).

Por fim, este trabalho mostra que $C$. pyramidalis sobrevive ao corte raso, independentemente da estação climática, num intervalo de tempo maior do que um ano. O período de três anos não é suficiente para recuperação da produção dos produtos madeireiros. É necessário um tempo de repouso maior para um novo ciclo de corte, visando manter a produção da espécie para atender à demanda energética da população rural.

\section{AGRADECIMENTOS}

À Empresa Pernambucana de Pesquisa Agropecuária (IPA) e à Comunidade de Caroalina, pela concessão de área e pelo apoio logístico para a implantação do experimento nas suas áreas, nos Municípios de Sertânia e Serra Talhada, e pelo apoio logístico; à Clothworkers Foundation (United Kingdom), por apoiar financeiramente a realização desta pesquisa; ao Dr. José Antônio Aleixo da Silva, pelo apoio na determinação do número mínimo representativo de plantas para realização do estudo; a Elizandro Souza, estagiário da APNE, pela digitação dos dados de campo; ao José Roberto de Almeida Monteiro (Sertânia) e Antônio de Souza Silva (Serra Talhada), juntamente com sua equipe, pelo apoio na coleta dos dados; e ao CNPq, pela concessão da bolsa-produtividade.

\section{REFERÊNCIAS}

ALCOFORADO-FILHO, F. G.; SAMPAIO, E. V. S. B.; RODAL, M. J. N. Florística e fitossociologia de um remanescente de vegetação caducifólia espinhosa arbórea em Caruaru, Pernambuco. Acta Botanica Brasílica, v. 17, n. 2, p.287-303, 2003.

ANALYTICAL SOFTWARE, 2000. Statistix 7.0 for Windows.

ANDRADE-LIMA, D. The caatinga dominium. Revista Brasileira de Botânica, v.4, p.149-153, 1981.

ANDRADE, L. A. et al. Análise da cobertura de duas fitofisionomia de caatinga, com diferentes históricos de uso no município de São João do Cariri, estado da Paraíba. Cerne, v.11, n.3, p.253-262, 2005.

ANDREIS, C. et al. Estudo fenológico em três fases sucessionais de uma floresta estacional decidual no município de Santa Tereza, RS, Brasil. Revista Árvore, v.29, n.1, p.55-63, 2003.

AMORIM, I. L.; SAMPAIO, E. V. S. B.; ARAÚJO, E. L. Flora e estrutura da vegetação arbustivo arbórea de uma área de caatinga do Seridó, RN. Acta Botanica Brasilica, v.19, p.615-623, 2005.

ARAÚJO, E. L.; SAMPAIO, E. V. S. B.; RODAL, M. J. N. Composição florística e fitossociologia de três áreas de caatinga de Pernambuco.

Revista Brasileira de Biologia, v.55, p.596-607, 1995.

R. Árvore, Viçosa-MG, v.32, n.6, p.1041-1049, 2008 
ARAÚJO, E. L.; TABARELLI, M. Estudos de ecologia de populações de plantas do nordeste do Brasil. In: ARAÚJO, E. L. et al. (Eds.).

Biodiversidade, conservação e uso sustentável da flora do Brasil. Recife: SBB/UFRPE, 2002. p.135-142.

ARAúJO, E. L.; FERRAZ, E. M. N. Processos ecológicos mantenedores da diversidade vegetal na caatinga: estado atual do conhecimento. In: CLAUDINO SALES, V. (Org.), Ecossistemas brasileiros: manejo e conservação. Fortaleza: Expressão Gráfica, 2003. p.115-128.

ARAÚJO, E. L. Estresses abióticos e bióticos como forças modeladoras da dinâmica de populações vegetais da caatinga. In: NOGUEIRA, R. J. M. et al. (Eds) Estresses ambientais: danos e benefícios em plantas. Recife: Universidade Federal Rural de Pernambuco, 2005. p.50-64.

ARAÚJO, E. L.; MARTINS, F. R.; SANTOS, A. M. Establishment and death of two dry tropical forest woody species in dry and rainy seasons in northeastern brazil.. In: NOGUEIRA, R. J. M. C. et al. (Eds.). Estresses ambientais: danos e benefícios em plantas. Recife: Universidade Federal Rural de Pernambuco, 2005. p.76-91.

ARAÚJO, E. L.; ALBUQUERQUE, U. A.; CASTRO, C. C. Dynamics of the Brazilian Caatinga $-\mathrm{a}$ revision concerning the plants, environments and people. Functional Ecosystems and communities, v.1, p.15-29, 2007.

BORCHERT, R. et al. Environmental control of flowering periodicity in Costa Rican and Mexican tropical dry forests. Global Ecology and Biogeography, v.13, n.5, p.409-425, 2004.

\section{EUCLIDES, R. F. Manual de utilização do} Programa SAEG (Sistema para análises estatísticas e genéticas). Viçosa, MG: Universidade Federal de Viçosa, 1983. 59p.

FERRAZ, E. M. N.; RODAL, M. J. N.; SAMPAIO, E. V. S. B. Physiognomy and structure of vegetation along an altitudinal gradient in the semi-arid region of northeastern Brazil. Phytocoenologia, v.33, n.1, p.71-92, 2003.

R. Árvore, Viçosa-MG, v.32, n.6, p.1041-1049, 2008
FERRAZ, J. S. F.; ALBUQUERQUE, U. P.; MEUNIER, I. M. J. Valor de uso e estrutura da vegetação lenhosa às margens do Riacho do Navio, Floresta, Pernambuco. Acta Botanica Brasilica, v.20, n.1, p.1-10, 2006.

FIGUEIRÔA, J. M. et al. (Eds.) Espécies da flora nordestina de importância econômica potencial. Recife: APNE, 2005. p.101-133.

FIGUEIRÔA, J. M. et al. Effects of cutting regimes in the dry and wet season on survival and sprouting of woody species from the semi-arid caatinga of northeast Brazil. Forest Ecology and Management, v.229, n.1/3, p.294-303, 2006.

FRANCELINO, M. R. et al. Contribuição da caatinga na sustentabilidade de projetos de assentamentos no sertão norte-rio-grandense. Revista Árvore, v.27, n.1, p.79-86, 2003.

LINAREZ-PALOMINO, R.; ALVAREZ, S. I. P. Tree community patterns in seasonally dry tropical forests in the Ceros de Amotape Cordillera, Tumbes, Peru. Forest Ecology and Management, v.229, p.261-272, 2005.

LUOGA, E. J.; WITKOWSKI, E. T. F.; BALKWILL, K. Regeneration by coppicing (resprouting) of miombo (African savanna) trees in relation to land use. Forest Ecology and Management, v.189, 1/3, p.23-35, 2004.

MANTOVANI, M. et al. Fenologia reprodutiva de espécies arbóreas em uma formação secundária da floresta Atlântica. Revista Árvore, v.27, n.4, p.451-458, 2005.

McCRAY, J. K.; WALSH, B.; HAMMETT, A. L. Species, sources, seasonality, and sustainability of fuelwood commercialization in Malaya.

Forest Ecology and Management, v.205, p.299-309, 2005.

McLAREN, K. P.; McDONALD, M. A. Coppice regrowth in a disturbed tropical dry limestone forest in Jamaica. Forest Ecology and Management, v.180, n.1/3, p.99-111, 2003.

MIRSA, M. K. et al. Domestic fuel energy consumption in an Indian urban ecosystem. Biomass and Bioenergy, v. 6, n. 9, p.473-486, 1995. 
MONTEIRO, J. M. et al. Teor de taninos em três espécies medicinais arbóreas simpáticas da caatinga. Revista Árvore, v.29, n.6, p.999-1005, 2005.

MORELLATO, L. P. C. et al. Phenology of Atlantic rain forest trees: a comparative study.

Biotropica, v.32, n.4b, p.811-823, 2000.

NEGREROS-CASTILLO, P.; HALL, R. B. Sprouting capability of 17 tropical tree species after overstory removal in Quintana Roo, Mexico.

Forest Ecology and Management, v.126, n.3, p.399-403, 2000.

NORDH, N. E.; VERWIJST, T. Above-ground biomass assessments and first cutting cycle production in willow (Salix sp.) coppice $-\mathrm{a}$ comparison between destructive and nondestructive methods. Biomass and

Bioenergy, v.27, n.1, p.1-8, 2004.

SAMPAIO, E. V. S. B. et al. Regeneração da vegetação da caatinga após corte e queima em Serra Talhada, PE. Pesquisa Agropecuária Brasileira, v.33, n.5, p.621-632, 1998.

SANTANA, J. A. S.; SOUTO, J. S. Estrutura e diversidade fitossociológica da caatinga na Estação Ecológica do Seridó-RN. Revista de Biologia e Ciência da Terra, v.6, n.2, p.232-242, 2006.
SANTOS, J. P.; ARAÚJO, E. L.; ALBUQUERQUE, U. P. Richness and distribution of useful woody plants in the semi-arid region of northeastern Brazil. Journal of Arid Environments, v.72, n.5, p.652-663, 2008.

SNEDECOR, G. W.; COCHRAN, W. G.

Estatistical methods. 6.ed. Ames: The Iowa State University Press, 1967. 593p.

TEWARI, S. K. et al. Effect of age and season of harvesting on the growth, coppicing characteristics and biomass productivity of Leucaena

leucocephala and Vitex negundo. Biomass and Bioenergy, v.26, n.3, p.229-234, 2004.

VALE, A. T.; FELFILI, J. M. Dry biomass distribution in a cerrado sensu stricto site in Brazil central. Revista Árvore, v.29, n.5, p.661-669, 2005.

VIRGINIO, J. F.; PAREYN, F. G. C. Situação da cobertura florestal no Nordeste. In: SAMPAIO, E. V. S. B. et al. Vegetação \& Flora da Caatinga. Recife: APNE, 2002.p.41-68.

ZAKIA, M. J. B. et al. O consumo de energéticos florestais no Rio Grande do Norte - Brasil. Natal: Projeto PNUD/FAO/ IBAMA, 1990. 40p. (Documento de Campo, 1)

ZAR, J. H. Biostatistical analysis. 3.ed. New Jersey: Prentice Hall, 1996. 662p. 